\title{
ESTRATEGIAS OPERATIVAS EN LA RESOLUCIÓN DE PROBLEMAS MATEMÁTICOS EN EL CICLO MEDIO DE LA E.G.B.
}

\author{
ALONSO García, v.', GONZÁlEZ CARMONA, A. ${ }^{2}$, SÁENZ BARRIo, $0 .^{3}$ \\ (1) Departamento de Didáctica de la Matemática de la Universidad de Cantabria. \\ (2) Departamento de Estadística e Investigación Operativa de la Universidad de Granada. \\ (3) Departamento de Didáctica y Organización Escolar de la Universidad de Granada.
}

\section{SUMMARY}

This work is the result of a research about how children really solve mathematical problems. Twelve strategies have been identified in problems of a very different nature.

\section{REVISIÓN DEL TEMA}

"Con la misma confianza, pompa y circunstancia concedidas a cosas tales como la maternidad, el pastel de manzana y la sopa de pollo (ésta última sólo para resfriados), se considera a la resolución de problemas como la innovación más importante de la matemática en la década de los 80\%, (Brown, 1983). Aunque afirmación tan rotunda queda suavizada por el tono festivo de la declaración y la prematuridad con que fue hecha, hoy podríamos ratificarla con la perspectiva que proporciona el casi final de la década. La publicación casi simultánea del Anuario de 1980 de la Asociación Nacional de Profesores de Matemáticas de Estados Unidos (NCTM), y el documento de la misma Asociación sobre una propuesta para la enseñanza de la matemática, puede ser considerada como el pistoletazo de saliđa para una acción defínida por el NCTM de la siguiente forma:

«El Consejo Nacional de Profesores de Matemáticas recomienda que la solución de problemas sea el principal objetivo de la enseñanza de las matemáticas en las escuelas de los ochentary.

Ello no obsta para que la resolución de problemas no haya sido con anterioridad objeto de controversia didáctica y haya rendido fructiferas lineas de investigación (Schoenfeld, 1985; Barnett, Vos, Sowder, 1979). De todas estas líneas, han ido reduciendo su producción las relativas a los aspectos materiales (formulación del enunciado, ayudas visuales, tamaño de las cantidades numéricas), verbales (complejidad sintáctica, lecturabilidad, posición de las claves verbales), contextuales (familiaridad, originalidad, realismo), para cen- trarse casi exclusivamente en el estudio de las variables relativas al proceso de resolución. Cabe señalar, sin embargo, que, a pesar de que las variables de proceso fueron consideradas ya desde el primer cuarto de este siglo como un campo relevante de la investigación sobre resolución de problemas, el concepto mismo de proceso estaba poco definido; por ejemplo, Barnett, Vos y Sowder señalan cómo muchos investigadores se centraron en el significado de las operaciones fundamentales o en seguir el desarrollo conducente a la correcta solución, por más que tales visiones del proceso sean parientes cercanas de la variable producto.

Hay práctica unanimidad al afirmar que la verdadera investigación sobre las variables de proceso se inicia a partir de los escritos de Polya, sobre todo con la aparición en 1945 de su How to solve it.

\section{MODELOS DE PROBLEM-SOLVING}

Lo que realmente aporta Polya es un modelo. Si la investigación precedente se focalizaba en ciertos aspectos materiales y concretos del enunciado, o en las hav bilidades específicas de los buenos/malos resolutores, bastaban sencillos instrumentos de codificación y medida para su análisis. Sin embargo la complejidad del proceso de solución de problemas requería procedimientos que permitieran el seguimiento y valoración de las distintas fases implicadas y, consecuentemente, de las habilidades o competencias requeridas. La heurística multifase de Polya subviene a esta necesidad, pero sobre todo ofrece un modelo formal tanto para 
quien resuelve problemas como para quien enseña a resolverlos. Las cuatro fases de Polya (1. Comprender el problema; 2 . Concebir un plan; 3 . Ejecutar el plan; 4. Examinar la solución obtenida) hacen referencia a «las operaciones mentales típicamente útiles» (p. 103 de la versión castellana de 1965). Para hacer más operativo el proceso de solución o de enseñanza, Polya desgrana cada estrategia general en varias subfases u operaciones (tres en la primera, ocho en la segunda, cinco en la cuarta) que estructuran el armazón heurístico de su método. Todavia el modelo puede verse aplicado en sus propios términos en obras relativamente recientes (p.e. Billstein, Libeskind y Lott, 1984).

La multiplicación de los modelos de solución de problemas consituye hoy, más que riqueza científica, una dispersión estéril de los esfuerzos en la investigación; unos porque añaden poco a la heurística de Polya (p.e., Schoen y Oehmke, 1980; Lee, 1982); otros porque no están suficientemente probados (p.e. Bransford y Stein, 1986; Fielker, 1985; Rudnick y Krulick, 1982), y aún los hay que son confusos en sus propuestas de «estrategias generales» y "estrategias de ayuda» (Charles, 1982). El modelo de Schoenfeld (en sus dos versiones de 1979 y 1982) con ser el más completo adolece, desde nuestro punto de vista, del mismo problema que afecta a todos los modelos que hemos revisado (y que podrían ser muchos más a la vista de los listados de «dissertations» para M. Ed., Ed. D. y Ph. D., tanto en educación como en enseñanza de las matemáticas en los últimos años). Tal dificultad consiste, repetimos, a nuestro modo de ver, en que son modelos formales construidos, a expensas de un a priori, que es el proceso ideal, conceptual, o lógico, si se quiere, para resolver problemas. En otros términos, son modelos a través de los cuales se factualiza una teoría. Y aquí no tenemos más remedio que estar en desacuerdo con Lester (1982) cuando afirma que tanto la práctica como la investigación sobre la solución de problemas ha carecido de una teoría. Que haya carecido de una teoria adecuada puede admitirse a la vista de los resultados contradictorios de la investigación, pero que no haya estado apoyada en algún tipo de teoría es falso.

Comprendemos por donde va Lester en las tres declaraciones relativas a la necesidad de la teoria, pero debemos hacer notar que en los modelos al uso subyacen al menos dos teorias. Una implícita, pero que dirige el proceso de pensamiento, y que es la heurísticodeductiva, propia del razonamiento matemático. Esta teoria, inspiradora de los métodos de enseñanza al uso, ha sido contundentemente criticada por Brown (1983) y Schoenfeld (1985b). Para el primero, la enseñanza de la resolución de problemas está viciada por la propia deformación profesional del matemático para quien, acostumbrado a demostrar que $p \rightarrow q$, lo único importante es la congruencia del proceso, valioso por sí mismo, y en el que tanto la verdad de $p$, como la personalidad, la cultura, el estilo cognitivo del investigador, son irrelevantes (p. 27 y 33 ). Schoenfeld viene a decir lo mismo con otras palabras: «aungue parezca raro, (nuestros alumnos) son las víctimas de nuestro profesionalismo, ... les presentamos los resultados de nuestras exploraciones matemáticas de manera orga * nizada y coherente's (p. 32).

La otra teoría, absolutamente explicita en los modelos de "problem-solving», es la que informa el paradigma didáctico llamado de proceso-producto, puesto que su objetivo expreso es mejorar las habilidades implicadas en el proceso a la espera de una mejora correlacional en la eficiencia resolutiva.

Por otra parte los modelos conceptuales analizados por Lesh et al. (1983) responden, es buena medida, a constructos teoréticos sobre manejo y procedimiento de información, lo cual parece apoyar la idea de una teoría cibernética del aprendizaje, explícitamente señalada por el Grupo Cero al establecer el paralelismo entre los enunciados de Polya en las etapas de programación en el uso de ordenadores (Grupo Cero, 1987).

En cualquier caso, el papel de los modelos didácticos, más que un programa rigido de entretenimiento, -que en definitiva la propuesta de los defensores del «modelling» (Lesh, Niss y Leo, 1986) - debería ser, en términos parecidos a lo que es el diseño curricular, una herramienta analítica en manos del profesor para juzgar la adecuación de sus estrategias y las de sus alumnos, así como un instrumento para transformar las «clarividencias» teóricas en definiciones prácticas para el aula (Howson y Malone, 1986).

\section{DEBILIDAD DE LOS MODELOS TEÓRICOS}

Que existe teoría es un hecho. Lo que ocurre es que solamente podrá acreditarse científicamente el modelo explicitador de una teoría cuando queden sólidamente establecidas las relaciones causaies entre el sistema y su modelo funcional. Y esto, desgraciadamente, no está suficientemente probado en la resolución de probiemas, por más que en una declaración voluntarista el Grupo de Comptencias Básicas del Instituto Nacional de Educación (EE.UU.) afirmara en 1975: «Los problemas se resuelven más eficazmente mediante la aplicación de una teoría adecuada». Y ésta es la que echa en falta Lester, de un lado porque no existe una única manera "correcta» de enseñar a resolver proble. mas y hay tantas maneras de enseñar eficazmente a pensar matemáticamente como profesores de talento haya (Schoenfeld, 1985a). De otro lado, porque la relación entre los procedimientos heurísticos descritos y la solución de problemas «depende más del análisis conceptual resiltante de una reflexión personal y del análisis de la propia experiencia en matemáticas que de un estudio de naturaleza estrictamente empirica" (Brown, 1983). Y sigue diciendo Brown: "La mayor parte del trabajo empírico surge de conceptualizaciones de na- 
turaleza teórica (ya sea articulada como tal, o no)») (p. 24). El hecho es que el trabajo empirico no termina de confirmar la validez de los constructos teóricos; p.e., hace ya más de diez años que Silver (1977) confirmó que el segundo heurístico de Polya, «trazar un plan», era más un aspecto o forma de reestructurar los datos del problema que de resolverlo. Otras investigaciones han revelado que los estudiantes varían el modelo formal de solución de un problema a otro; que hay contradicción entre el número y variedad de estrategias utilizadas por los buenos y deficientes resolutores de problemas; que el uso del ensayo y error, identificado en algunos estudios como característico de los malos resolutores, se encuentra, en otros casos, por encima de la media en las tentativas de los buenos, y que además, el ensayo y error conduce al éxito con más frecuencia que el uso de la deducción (Barnett, Vos y Sowder, 1979).

En definitiva, en contra de la opinión de que el uso de modelos es un buen método de enseñanza para la resolución de problemas, las conclusiones de la investigación son inconsistentes. Por ejemplo, la conclusión de Lesh y sus colaboradores es que las técnicas que los estudiantes aprenden en el colegio no se tranfieren al mundo real y que los modelos generales no se aplican a situaciones especificas (Lesh, Landau y Hamilton, 1983). Por su parte Schoenfeld afirma: «El pensamiento matemático es lógico y riguroso, y las técricas que empleamos (los matemáticos) para enfrentarnos a los problemas son aplicables en muchos campos, pero a menos que las presentemos con claridad, no es probable que los alumnos adquieran la intuición necesaria para 'comprender' o beneficiarse de ellas" (1985b).

Con muy pocas excepciones la instrucción en un modelo de solución se ha mostrado poco eficaz en el desarrollo de la capacidad general para resolver problemas. Por el contrario, la enseñanza de procedimientos específicos utilizados en el proceso de resolución, tales como representar mediante dibujos, confeccionar esquemas, clasifficar datos, construir algoritmos, etc... incremen taba su uso en la resolución o mejoraba el enfoque de problemas en cuyo enunciado se utilizaba alguno de tales recursos, pero la mejora de la capacidad general resolutiva era muy modesta.

Por otro lado, la variada categorización de procesos, heuristicos, estrategias, fases, etc... tanto en número como en la definición de la conducta exhibida o impljcada, dificulta la convergencia hacia un modelo de integración. Desde el modesto esquema analítico de Means y Loree (1986) con tres variables hasta las diecisiete de Flaherty (1975), el de Kantowski (1977) con siete, hasta el último de Schoenfeld (1982) con veintisiete, entre estrategias generales, estrategias básicas y actividades especificas, además de los modelos citados anteriormente, uno puede hacerse una idea del estado en que se encuentra hoy la investigación en este campo de los modelos propositivos.

\section{NUESTRO PUNTO DE PARTIDA}

El desconcierto por la inconsistencia de los resultados de la investigación difícilmente puede ser resuelto desde dentro del mismo paradigma. El modelo de caja negra, que está en la base del paradigma procesoproducto, deja sin explicar lo que ocurre dentro de la caja; «sólo estudia concomitancias entre entradas y salidas del sistema; al no haber hilos de explicación no hay posibilidades de orientación racional de nuevos ensayos ante el fracaso" (Gimeno, 1981, p. 50). De ahí que la instrucción de modelos se haya mostrado más productiva en habilidades especifficas que en el complejo proceso de producción; por esta razón, nuestro trabajo no pretende cuantificar las respuestas correctas, -al menos no es ése el objetivo central - , sino determinar qué tipo de competencias, habilidades o conductas utilizan espontáneamente los escolares, y si es posible identificar algún patrón estable que pueda considerarse como un estilo de resolución de problemas.

Ante esta situación cabía preguntarse: ¿Cuál es la conducta típica de un escolar ante los problemas, su «individual's own approachn? Dado que las formas de comportamiento teóricamente posibles son casi infinitas y que, además, constituyen un campo abierto en que pueden aparecer elementos inéditos que lo amplian y complican, podria intentarse una respuesta que describiera, dentro de un cierto margen de probabilidad, el esquema heurístico de un alumno o de un grupo, y permitiera poner en relación teoría y práctica sobre un soporte experiencial.

Esta idea está muy próxima a la que Verstappen expuso en el grupo de estudio sobre «Investigación y Enseñanzas en la quinta reunión del ICME (1984) cuando afirmaba que el objetivo supremo para los profesores era identificar alguna suerte de estructura que permitiera apoyar cambios en la acción pedagógica de forma que se pudiera penetrar en los insights matemáticos de los estudiantes, y estimular al profesor como investigador en el aula. (Christiansen et al., 1986).

E\} método de elección para este tipo de trabajos parece ser el de la entrevista clínica. Esta técnica ya la habia utilizado Wittman en los programas para la formación de profesores de Dortmund. En su comunicación a la misma mesa del citado congreso, dice Wittman que sla entrevista clínica es el camino más fácil de hacer psicología, promover actitudes y competencias docentes, y relacionar la psicología con otros dominios de la formación de profesores de matemáticas» (p. 285).

Sobre estas premisas discurre este trabajo.

\section{CATEGORIZACIÓN}

Para ello, se ha partido de un conjunto de conductas que los niños suelen mostrar reiteradamente en la re- 
solución de problemas. Tales actos prototípicos, reducidos a los que un equipo de profesores de E.G.B. ha estimado como operantes significativos, son los doce siguientes:

$E_{1}=$ Lectura del problema

$\mathrm{E}_{2}=$ Escritura de datos

$\mathrm{E}_{3}=$ Representación simbólica de los datos (dibujos, gráficos, tablas).

$\mathrm{E}_{4}=$ Utilización de datos irrelevantes

$E_{s}=$ Perplejidad: sentirse desconcertado, repetir la pregunta del problema, repetirse los datos o pedir más. $\mathrm{E}_{6}=$ Formulación de hipótesis (si hago esto, entonces...) y planes de trabajo.

$\mathrm{E}_{7}$ = Anticipación de los resultados (estimación cuantitativa o cualitativa).

$\mathrm{E}_{\mathrm{B}}=$ Cálculo (operaciones)

E9 = Resolución (llegar a un témino en el proceso operativo y dar una solución)

$E_{10}=$ Comprobación (verificar que el resultado satisface las condiciones)

$E_{11}=$ Rectificación (cambiar de estrategia: Orden operacional, simplificar el problema, utilizar otros datos)

$E_{12}=$ Estancamiento o ausencia de conducta.

Definido así el campo de observación ya puede someterse a experimentación. En principio todas estas actividades son igualmente posibles y mutuamente excluyentes, es decir, un sujeto no puede ser observado simultáneamente en dos o más de ellas. A partir de estas condiciones podemos preguntarnos: $i$ cual es la probabilidad de que un determinado escolar proceda de un cierto modo?; ¿cuál es la probabilidad de que a un acto concreto siga otro? Se trata, pues, de investigar las conductas típicas de los escolares frente a los problemas matemáticos, ya que a esta edad el niño ha alcanzado la madurez suficiente para enfrentarse a ellos con una variedad material y formal que prefigura tareas más complejas y extensas.

\section{MUESTRA}

Se partió de una muestra constituida por 102 niños de $5^{\circ}$ curso de E.G.B. de 4 colegios situados en cada uno de los puntos cardinales de la ciudad de Santander.

\section{CONTENIDO}

Por lo que se refiere al contenido, se establecieron 5 áreas: conjuntos, numeración, cólculo, magnitudes y geometría. Se pidió a los profesores de esos centros encargados de la enseñanza de las matemáticas que eligieran 4 problemas standard de cada área correspondientes a los cursos $3^{\circ}, 4^{\circ}$ y $5^{\circ}$ de E.G.B. Se obtuvieron así 80 enunciados, que se sometieron a sorteo con bolas numeradas hasta recoger la muestra que expone el cuadro adjunto.

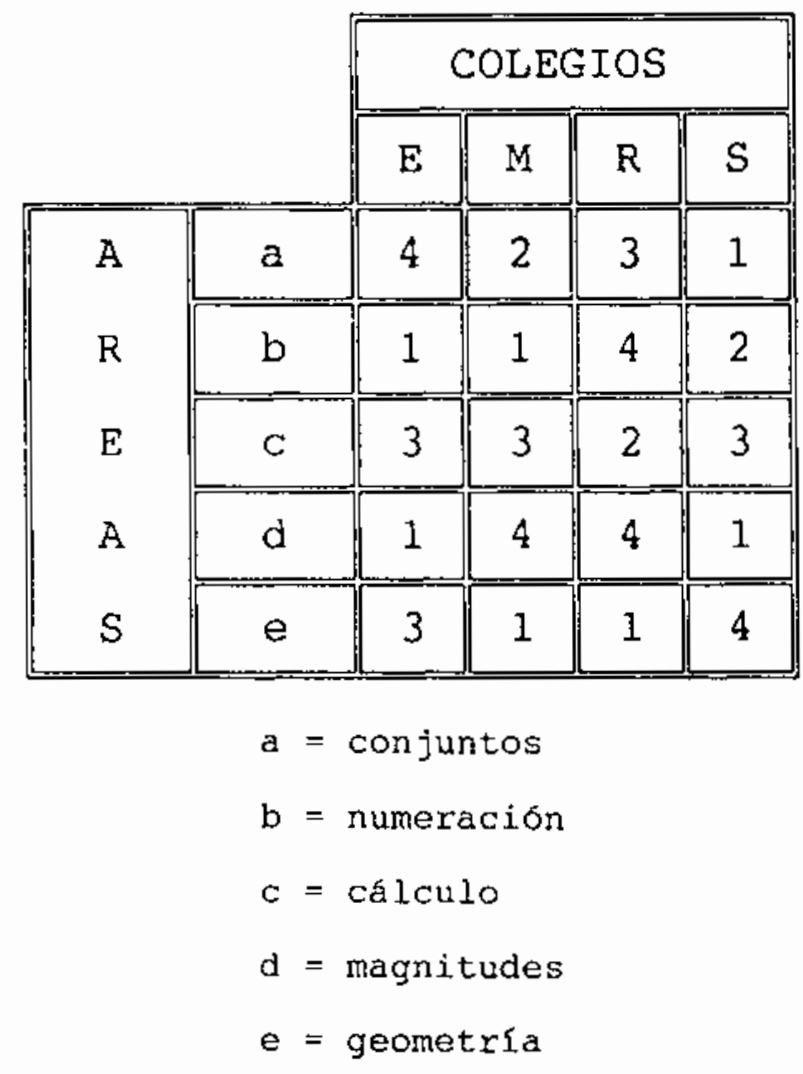

Las cifras indican el número de orden de cada área, en la relación que facilitó cada uno de los colegios.

\section{PREPARACIÓN}

Estos 20 problemas se presentaron para su resolución a los 102 niños de la muestra. De los 20 problemas se seleccionaron para su aplicación final 5 problemas, uno por cada área matemática, y que hubieran obtenido en la aplicación piloto entre un $66 \%$ y un $75 \%$ de resultados positivos. La reducción a cinco problemas era exigible por la laboriosidad de la aplicación, que no permitía una observación muy dilatada. Y el porcentaje de éxitos, porque se entendió que eran problemas idóneos del ciclo, ni muy fáciles ni muy difíciles, sino representativos de las habilidades características en matemáticas de los alumnos típicos del ciclo medio. (Estos problemas, se incluyen como apéndice al final del articulo).

Dada la meticulosidad de la técnica de observación, la complejidad del sistema de computación de conductas y la cantidad de tiempo necesario para el registro individual, era imprescindible reducir el número de casos, que quedó establecido en 20 niños. Esta reducción debia hacerse sin pérdida de representavidad, que se calculó mediante un contraste de medias, previa la comprobación de la normalidad de las distribuciones, llegando a la conclusión de que efectivamente, eran dos 
estimaciones de la media de una misma población (Alonso, 1986).

Elegidos al azar 5 niños de cada uno de los mismos colegios, aulas y grupo que habian participado en la primera fase, se decidió estudiar su conducta en el curso de la resolución de los problemas de modo directo y simultáneo con registros personales, mediante parejas de observadores, debidamente adiestrados, y registros auditivos (magnetófono) y visuales (videógrafos). Calculamos la fiabilidad de cada dos series de observaciones hasta conseguir que el coeficiente fi de Scott fuese mayor que 0.85 , indice aceptable en la investigación pedagógica.

\section{REALIZACIÓN}

Los niños pensaban en voz alta expresando todas sus vivencias en el curso del proceso de resolución. Exteriorizaban verbalmente su proceder sin la menor dificultad e incluso de una forma mucho más natural de lo esperado. Las parejas de examinadores-observadores anotaban la serie de las conductas exhibidas en intervalos de 15 segundos en protocolos que recogen el curso de la conducta de cada niño ante cada problema, mediante un sistema de notación que comprende números del 1 al 12, según los subíndices correspondientes a cada una de las estrategias.

\section{TRATAMIENTO}

Aunque se utilizó un sistema de registro estático, los datos se codificaron de forma dinámica, referida, asimismo, a niño y problema, mediante matrices de transición, que permiten descubrir en una conducta seriada si la sucesión de los acontecimientos surge al azar, o por el contrario hay una cierta tendencia, medida en términos de probabilidad, de que un suceso ocurrido en un tiempo, proceda de otro, y de que en la unidad temporal siguiente permanezca en la misma situación o pase a otra.

El tratamiento de las observaciones se ha realizado mediante la aplicación de un algoritmo para cadenas Mar- kovianas estudiado por González Carmona (1975) y cuya eficacia ya se ha probado en la descripción de conductas cuya variable fundamental, más que la mera ocurrencia del evento, es la transición de un estado a otro en una secuencia tempora! (Francés, González y Sáenz, 1987 y Francés y Sáenz, 1987).

De cada sujeto se obtuvieron 12 matrices de transición 12 por 12 (2 por cada problema en términos de frecuencia: $\left(n_{i, j}\right)$, y en términos de probabilidades: $\left(p_{i, j}\right)$, así como otras 2 más de la misma forma que engloban los cinco problemas); además 10 nuevas matrices: 5 de ellas exponen de forma global las frecuencias $n_{i, j}$ de transición referentes a los resultados de cada problema de los 20 niños en su conjunto y las otras 5 las probabilidades de transición con sus grafos respectivos; 2 úttimas matrices: una que resume las frecuencias de transición totales y la otra expresa las probabilidades con que es posible esperar esas transiciones. Cada una de las matrices contiene doce estados $E_{j}$.

Las matrices de frecuencias absolutas de transición $\left(n_{i, j}\right)$ vienen definidas de acuerdo con la condición de que cada elemento $n_{i, j}$ sea el número de transiciones observadas desde el estado $E_{i}$ al $E_{j}: n_{i, j}=\left(E_{i}, E_{i}\right)$.

Esta matriz, nos ofrece una información mucho más rica que la simple exposición de las frecuencias absolutas, aunque éstas se pueden obtener, si se quiere, mediante la suma de los términos $n_{1, j}, n_{2, j}, n_{3, j}, \ldots n_{12, j}$, de la columna $\mathrm{j}$ :

$$
f\left(E_{1}\right)=\sum_{i=1}^{12} n_{i}, 1 ; \ldots \ldots . ; f\left(E_{12}\right)=\sum_{i=1}^{12} n_{i} i_{2}
$$

En la tabla 1 se ofrece, por su valor informativo, un resumen de las frecuencias absolutas de los 20 niños en los 5 problemas, y las frecuencias relativas globales de cada conducta.

\section{EXPLOTACIÓN SIMPLE DE LOS DATOS}

Las matrices ofrecen la misma información que el cálculo tradicional, y además información adicional que

Tabla 1

\begin{tabular}{rrrrrrrrrrrrr}
\hline & $E_{2}$ & $E_{2}$ & $E_{3}$ & $E_{4}$ & $E_{5}$ & $E_{6}$ & $E_{7}$ & $E_{8}$ & $E_{9}$ & $E_{20}$ & $E_{22}$ & $E_{22}$ \\
$P_{2}$ & 254 & 78 & 0 & 2 & 211 & 21 & 7 & 63 & 12 & 0 & 6 & 111 \\
$P_{2}$ & 227 & 143 & 94 & 47 & 309 & 54 & 17 & 245 & 54 & 0 & 21 & 54 \\
$P_{3}$ & 190 & 113 & 0 & 1 & 77 & 40 & 1 & 499 & 41 & 5 & 69 & 5 \\
$P_{4}$ & 224 & 182 & 0 & 22 & 296 & 101 & 5 & 368 & 84 & 7 & 38 & 37 \\
$P_{5}$ & 394 & 172 & 0 & 54 & 544 & 113 & 0 & 453 & 102 & 5 & 23 & 63 \\
& & 1289 & 688 & 94 & 126 & 1437 & $\frac{329}{30}$ & $\frac{1628}{293}$ & $\frac{17}{17}$ & 157 & 270 \\
$E_{2}$ & $E_{2}$ & $E_{3}$ & $E_{4}$ & $E_{5}$ & $E_{8}$ & $E_{7}$ & $E_{8}$ & $E_{9}$ & $E_{20}$ & $E_{22}$ & $E_{22}$ \\
12.89 & 6.88 & 0.94 & 1.26 & 14.37 & 3.29 & 0.30 & 16.28 & 2.93 & 0.17 & 1.57 & 2.70
\end{tabular}


las tablas ordinarias de frecuencia no pueden proporcionar.

Veamos a modo de ejemplo dos de las matrices individuales de la niña Alicia: Problema 4, tal como se han descrito (Tabla 2).

En el caso de Alicia, sus estrategias en la solución del problema 4 nos revelan que Eı (lectura del problema) fue utilizada 8 veces mientras que la matriz $\left(n_{i, j}\right)$ de frecuencias de transición de Markov nos informa que pasó de $E_{1}$ a $E_{1}, n_{l, 1}=7$ veces y con una probabilidad $p_{1,1}=0.9$ y que comenzó con la lectura sin haber utilizado previamente otra estrategia.

La $\mathrm{E}_{2}$ fue utilizada 14 veces. La matriz $\left(\mathrm{n}_{\mathrm{i}, \mathrm{j}}\right)$ nos informa que pasó de $E_{2}$ a $E_{2}, n_{2,2}=5$ veces con una probabilidad de transición de p2.2 $=0.30$ (de escribir datos a seguir escribiéndolos); de $\mathrm{E}_{4}$ a $\mathrm{E}_{2}, \mathrm{n}_{4,2}=3$ veces con una probabilidad de transición p $4,2=1$ (de utilizar datos irrelevantes a escribirlos); de $E_{s}$ a $E_{2,} n_{5,2}=$ 1 vez con una probabilidad $\mathrm{p} s, 2_{12}=0.04$ (de sentirse desconcertado a escribir datos); de $\mathrm{E}_{6} \mathrm{a} \mathrm{E}_{2, n 6,2}=4$ veces con $p_{6,2}=0.44$ (de formular hipótesis a escribir datos); de $\mathrm{E}_{9}$ a $\mathrm{E}_{2}, \mathrm{n}_{9,2}=1$ vez con $p_{9,2}=0.20$ (de conseguir un resultado a escribir de nuevos datos), etc...

Tabla II

Matriz de frecuencias de transicion

\begin{tabular}{|c|c|c|c|c|c|c|c|c|c|c|c|}
\hline & $E_{1}$ & $E_{2}$ & $E_{3}$ & $E_{4}$ & $E_{s}$ & $E_{E}$ & $E_{2}$ & $\varepsilon_{0}$ & $E_{9}$ & $E_{10}$ & $E_{12}$ \\
\hline$E_{1}$ & 7 & 0 & 0 & 0 & 0 & 1 & 0 & 0 & 0 & 0 & 0 \\
\hline$E_{2}$ & 0 & 5 & 0 & 0 & 1 & 1 & 0 & 6 & 0 & 0 & 0 \\
\hline$E_{3}$ & 0 & 0 & 0 & 0 & 0 & 0 & 0 & 0 & 0 & 0 & 0 \\
\hline$E_{4}$ & 0 & 3 & 0 & 0 & 0 & 0 & 0 & 0 & 0 & 0 & 0 \\
\hline$E_{s}$ & 0 & 1 & 0 & 0 & 20 & 4 & 1 & 0 & 0 & 0 & 0 \\
\hline$E_{G}$ & 0 & 4 & 0 & 1 & 3 & 1 & 0 & 0 & 0 & 0 & 0 \\
\hline $\mathrm{E}_{7}$ & 0 & 0 & 0 & 0 & 0 & 1 & 0 & 0 & 0 & 0 & 0 \\
\hline$E_{g}$ & 0 & 0 & 0 & 0 & 0 & 0 & 0 & 14 & 5 & 0 & 1 \\
\hline$E_{9}$ & 0 & 1 & 0 & 2 & 0 & 0 & 0 & 0 & 1 & 1 & 0 \\
\hline$E_{10}$ & 0 & 0 & 0 & 0 & 1 & 0 & 0 & 0 & 0 & 0 & 0 \\
\hline$E_{21}$ & 0 & 0 & 0 & 0 & 0 & 1 & 0 & 0 & 0 & 0 & 0 \\
\hline$E_{12}$ & 0 & 0 & 0 & 0 & 1 & 0 & 0 & 0 & 0 & 0 & 0 \\
\hline
\end{tabular}

\begin{tabular}{|c|c|c|c|c|c|c|c|c|c|c|c|c|}
\hline & & & & & & uen & ias & & & & & \\
\hline$f:$ & $\begin{array}{r}E_{1} \\
8\end{array}$ & $\begin{array}{l}E_{2} \\
14\end{array}$ & $\begin{array}{r}E_{3} \\
0\end{array}$ & $\mathrm{E}_{3}$ & $\begin{array}{l}E_{s} \\
26\end{array}$ & $\begin{array}{r}E_{6} \\
9\end{array}$ & $E_{7}$ & $\begin{array}{l}E_{\theta} \\
20\end{array}$ & $\begin{array}{r}E_{9} \\
8\end{array}$ & $\begin{array}{r}E_{2} \\
1\end{array}$ & $\mathrm{E}_{22}$ & $\begin{array}{r}E_{12} \\
1\end{array}$ \\
\hline & & & tri & de & cobab & lid & les & & 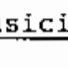 & & & \\
\hline & $E_{2}$ & $\mathrm{E}_{2}$ & $E_{3}$ & $\varepsilon_{a}$ & $E_{5}$ & $E_{6}$ & $E_{7}$ & $E_{\mathbf{a}}$ & $\mathrm{E}_{9}$ & $E_{20}$ & $E_{12}$ & $E_{12}$ \\
\hline$E_{2}$ & .87 & .00 & .00 & .00 & .00 & .1 & .00 & .00 & .00 & .00 & .00 & .00 \\
\hline & .0 & .36 & .00 & .0 & .07 & .07 & .00 & .4 & .00 & .00 & .00 & .07 \\
\hline 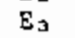 & .00 & .00 & .00 & .00 & .00 & .00 & .00 & .00 & .00 & .00 & .00 & .00 \\
\hline $\mathrm{E}_{\mathbf{A}}$ & .00 & 1.0 & .00 & .00 & .00 & .00 & .00 & .00 & .00 & .00 & .00 & .00 \\
\hline$E_{5}$ & .00 & .04 & .00 & .0 & .7 & .1 & .0 & .0 & .00 & .00 & .00 & .00 \\
\hline $\mathrm{E}_{\sigma}$ & .0 & .44 & .00 & .1 & 33 & .11 & .0 & .0 & .00 & .0 & .00 & .00 \\
\hline & .0 & .00 & .00 & 0 & .00 & 1.0 & .0 & .0 & .0 & .0 & .00 & .00 \\
\hline & .0 & .00 & .00 & .0 & .00 & .0 & .0 & .7 & .2 & .0 & .05 & .00 \\
\hline$E_{9}$ & .00 & .20 & .00 & .40 & .00 & .0 & .0 & .0 & .20 & .2 & .00 & .00 \\
\hline & .0 & .00 & .0 & .0 & 1. & .0 & .0 & & .00 & & .00 & .00 \\
\hline E & .00 & .00 & .00 & .00 & .00 & 1.0 & .0 & .0 & .00 & .0 & .00 & .00 \\
\hline$E_{12}$ & .00 & .00 & .00 & .00 & 1.0 & .00 & .00 & .00 & .00 & .00 & .00 & .00 \\
\hline
\end{tabular}


Estos datos no son solamente una descripción de las estrategias utilizadas por un sujeto determinado en la resolución de un problema concreto, sino que sirven realmente como un instrumento de diagnóstico que permite detectar las diferentes fases que recorre un sujeto en su proceso mental a la búsqueda de la solución. Este conocimiento es el único criterio que garantiza la racionalidad de un programa de reeducación o tratamiento de las dificultades, si las hubiera.

\section{MATRICES DE TRANSICIÓN Y VECTOR LÍMITE}

Puede decirse que la matriz $\left(n_{i, j}\right)$ de frecuencias de transición, da una visión de la versatilidad con que es utilizada cada una de las formas de conducta Ei y que no es posible averiguar a partir de los datos que proporciona la tabla de frecuencias.

Considerando la sucesión registrada en los estadillos de observación, y admitiendo que el conjunto de las doce estrategias $E_{i}$ satisface las dos propiedades siguientes:

i) Cada resultado de observación pertenece al conjunto finito de estrategias $E_{1}, E_{2}, E_{3} . E_{4}, E_{5}, E_{6}, E_{7}, E_{8} . E_{9}$ $E_{10}, E_{11}$. E12.; que podemos llamar espacio de estados del sistema. Si el resultado de la n-ésima observación es $\mathrm{E}_{\mathrm{i}}$ entonces decimos que el sistema está en estado $E_{i}$ en el paso n-ésimo.

ii) El resultado de una observación depende a lo sumo del resultado de la observación inmediatamente anterior y no de cualquier otro resultado previo. Con cada par de estados $\left(E_{i}, E_{j}\right)$ se establece la probabilidad $\mathrm{p}_{\mathrm{i}, \mathrm{j}}$ de que $\mathrm{E}_{\mathrm{j}}$ suceda inmediatamente después de que suceda $E_{i}$.

El proceso estocástico se puede insertar dentro de las llamadas CADENAS DE MARKOV (finitas).

Los números $p_{1, j}$ Ilamados probabilidades de transición, se pueden ordenar en una matriz

$$
P=\left(p_{i, j}\right)
$$

llamada Matriz de transición.

A cada $E_{i}$ corresponde el vector fila ( $p_{i, 1} p_{i, 2}, \ldots, p_{i, 12}$ ). $\mathrm{Si}$ el sistema está en el estado $\mathrm{E}_{\text {, }}$ entonces el vector $\mathrm{f} i$ la representa las probabilidades de todos los estados posibles de la observación siguiente.

A partir de las matrices de frecuencias de transición absolutas $\left(n_{1,}\right)$ podemos obtener unas matrices estimadas $P^{*}=\left(P^{*}{ }_{i, j}\right)$, normalizando la matriz de frecuencias, al dividir cada elemento $n_{i, j}$ por la suma de los elementos de su misma fila:

asi de $\left(\mathrm{n}_{\mathrm{i}, \mathrm{j}}\right)$ se obtiene $\left(\mathrm{p}_{\mathrm{i}, \mathrm{j}}\right)$.
Si un sujeto o un grupo de sujetos se encuentra en un estado $E_{i}$ con una probabilidad ni, estas probabilidades se pueden representar por el llamado vector de distribución de probabilidad. Podemos describir la distribución de probabilidad inicial por $\pi^{(0)}$ y por $\pi^{(n)}$ la distribución de probabilidad en el paso enésimo.

Hay dos tipos de vectores de particular interés: los vectores limites y los vectores estacionarios, pudiendo o no existir en cada caso concreto.

Un vector estacionario $V$ indica que en el caso de que el sistema (el proceso mental de un niño o del grupo) se represente por él, permanecerá en él indefinidamente.

Un vector límite $W$ corresponde al hecho de que, partiendo de cualquier posible representación del sistema y pasado cierto tiempo, se obtiene como limite el vector mencionado. Si este vector existe, entonces es estacionario y no existirán otros vectores distintos de él que sean estacionarios.

En todos los casos que hemos estudiado existen estos vectores límite por cumplir el criterio de existencia de comunicación entre los estados y de un ciclo unidad (González Carmona, ibídem).

La existencia del vector límite implica que sea cual sea la situación inicial (en nuestro caso un proceso mental individual o de grupo), llegará a alcanzarse el vector límite, por lo que estaremos en una situación de equilibrio. Lo que significa que los procesos iniciales de un sujeto o de un grupo son irrelevantes, ya que con mayor o menor rapidez se irán acercando hasta exhibir las condiciones representadas por el vector límite.

Se ha encontrado que nuestras matrices de transición $P^{(n)}$ llegan a estados estacionarios en la potencia $\mathrm{n}=32$. A partir de esta potencia todas sus filas son iguales y el vector de distribución de probabilidad $\pi^{(32)} \mathrm{re-}$ presenta el vector límite W.

\section{POSIBILIDADES DEL CALCULO}

Tanto desde el punto de vista descriptivo como de diagnóstico, el tratamiento matricial de datos de observación es muy versátil. Con anterioridad se ha presentado la matriz de transición de un sujeto en un problema. Pero la propiedad aditiva de las matrices permite obtener una nueva que sea el resultado de la combinación de varias matrices singulares. De esta suerte se puede obtener:

a) Una matriz que refleje el perfil heuristico de un sujeto en la solución de un conjunto de problemas, lo que equivaldría a la secuencia prototípica de dicho sujeto, en el uso de las estrategias analizadas, para cualquier tipo de problema. El conocimiento de dicho proceso individualizado permitiria una intervención personalizada.

b) La matriz de un problema a partir del uso que de 
las distintas estrategias hace un cierto número de escolares, lo que facilitaria descubrir qué transiciones, y en qué orden, son prioritariamente elegidas por una cohorte en diferentes tipos de problemas.

c) Una matriz global o «final» que sea el resultado del enfrentamiento de muchos niños con muchos problemas. Esta matriz reflejaria las transiciones probabilisticamente más frecuentes de la cohorte sea cual fuere el tipo de problema; es decir, qué estrategias o cadenas heurísticas son instrumentadas preferentemente por un grupo de edad, nivel escolar, etc...

\section{RESULTADOS}

\subsection{Perfiles individuales}

La suma de matrices de un sujeto permite descubrir su estilo personal de ataque y desarrollo de los problemas; algo asi como su perfil heurístico o metodologia resolutiva personal. Siguiendo con Alicia, he aquí cómo utiliza sus recursos mentales y operativos en el conjunto de los cinco problemas resueltos:

Las 412 conductas registradas a Alicia en la resolución de los cinco problemas se han distribuido de la forma que indica la tabla 3.

El mayor número de transiciones lo registra $\mathrm{E} s,_{s} \tan$ to de recepción como de partida, pero sobre todo cabe destacar el carácter absorbente de este estado, en que la niña es observada durante largos períodos de tiempo $o$ al que retorna desde cualquier otra actividad. A continuación aparecen $E_{8} E_{2}$ y $E_{6}$, por este orden (aparte de la mera lectura), lo que evidencia que Alicia se centra con preferencia en los aspectos numéricos más que en estrategias de descubrimiento. La alta incidercia en Esse explica desde el momento en que el abordaje es puramente por tanteo; en consecuencia, al no atacar los problemas mediante un plan organizado, las fases de perplejidad y de retomo se plantean cuantas veces los datos o los procedimientos de cálculo eleg;dos no parecen "razonables»; pero sin que el criterio de «razonabilidad» lo sea a su vez, por cuan to que Alicia opera al azar (ver tabla 4).

El primer contacto con el problema es la lectura. A partir de $E_{6}$ la cadena probabilistica se establece como indica el grafo 1.

A partir de la lectura del problema, la niña se estaciona, como parece natural, en una actitud aparentemente inquisitiva $\left(\mathrm{E}_{5}\right)$; y es aparente porque si fuese verdadera, la transición natural sería hacia $E_{6} \circ E_{7}$ con una probabilidad mucho mayor que la reflejada. El hecho de que permanezca en $E_{s}$, de forma circular y persistente, demuestra que la perplejidad no es racional sino verdadero bloqueo emotivo.

Tabta III

\begin{tabular}{rrrrrrrrrrrr}
\hline$E_{2}$ & $E_{2}$ & $E_{3}$ & $E_{4}$ & $E_{3}$ & $E_{6}$ & $E_{7}$ & $E_{8}$ & $E_{9}$ & $E_{10}$ & $E_{12}$ & $E_{22}$ \\
62 & 45 & 0 & 7 & 121 & 31 & 6 & 98 & 16 & 2 & 11 & 13 \\
\hline
\end{tabular}

Tabla IV

\begin{tabular}{|c|c|c|c|c|c|c|c|c|c|c|c|c|}
\hline$E_{1}$ & 83 & $\begin{array}{r}E 2 \\
.00\end{array}$ & $\begin{array}{l}\mathrm{E}_{3} \\
.00\end{array}$ & $\begin{array}{l}\mathbf{E}_{4} \\
.00\end{array}$ & $E$ & $\begin{array}{l}E_{6} \\
.02\end{array}$ & $\begin{array}{l}\mathrm{E}_{7} \\
.00\end{array}$ & $\begin{array}{l}E_{\mathbf{a}} \\
.00\end{array}$ & $\begin{array}{l}E_{9} \\
.00\end{array}$ & $\begin{array}{r}E_{20} \\
.00\end{array}$ & $\begin{array}{l}E_{21} \\
.00\end{array}$ & $\begin{array}{l}E_{22} \\
.02\end{array}$ \\
\hline$E_{2}$ & .00 & .3 & .00 & .00 & .09 & .07 & .02 & .41 & .0 & .0 & .00 & .02 \\
\hline$E_{3}$ & .00 & .0 & .0 & .0 & .0 & .00 & .00 & .0 &. & .0 & .00 & .00 \\
\hline$E_{4}$ & .00 & .5 & .0 & .0 & .2 & .0 & .00 & .12 & , & .1 & .00 & .00 \\
\hline$E_{5}$ & .03 & .0 & .0 & .0 & .62 & .1 & .0 & .0 & .8 & .0 & .03 & .05 \\
\hline $\mathrm{E}_{\bar{\sigma}}$ & .0 & .3 & .0 & .06 & .42 & .03 & .0 & .1 & .0 & .8 & .00 & .00 \\
\hline E, & .0 & .5 & .0 & .00 & 0 & .1 & .0 & .1 & .0 & .0 & .00 & .17 \\
\hline$E_{\theta}$ & .00 & .0 & .0 & .01 & .0 & .0 & .00 & .6 & .1 & .0 & .08 & .00 \\
\hline$E_{9}$ & .08 & .0 & .0 & .17 & .08 & .25 & .00 & .0 & & .0 & .00 & .00 \\
\hline$E_{0}$ & .00 & .0 & .0 & .00 & 1.0 & .0 & .0 & .00 & .0 & .0 & .00 & .00 \\
\hline$E_{1}$ & .0 & .0 & .0 & .00 & .2 & .1 & .0 & .4 & & .0 & & \\
\hline$E_{22}$ & .08 & .00 & .00 & .00 & .53 & .08 & 0 & .0 & .0 & .00 & .00 & \\
\hline
\end{tabular}

Grafo I

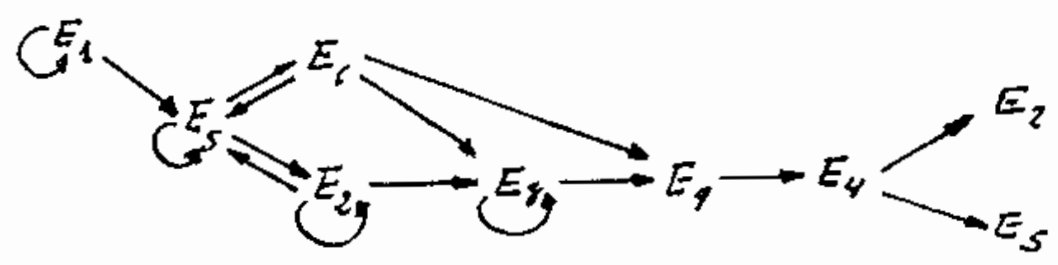


Alternativamente salta a $E_{2}$ (utilización de datos) y $E_{8}$ (cálculo), pero como su estrategia es «blind guessing», no hay comprobación, por lo que, sin justificación, pasa de la obtención de un resultado (Eq) a un anárquico uso por ensayo y error de datos irrelevantes $\left(E_{4}\right)$ con los que encontrar una solución más plausible, a pesar de que no haya justificado el posible error de los ya obtenidos. El desembarco en $\mathrm{E}_{4}$ significa volver a

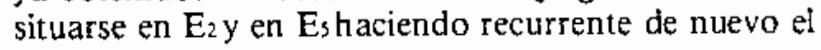
proceso.

Es evidente que Alicia requiere una intervención decisiva en dos momentos: 1) En $E_{5}$ para salir hacia $E_{6}$, mediante la producción de una hipótesis, de trabajo o plan de desarrollo, y pasar a continuación hacia $\mathrm{E}_{7}$; y 2) En Eq que sólo tiene una salida razonable que es hacia $E_{10}$, con una bifurcación: hacia $E_{11}$ para reestructurar el proceso o hacia $\mathrm{E}_{12}$, estado de reposo y refuerzo, por haber resuelto el problema.

\subsection{Matriz de problema-tipo}

El problema 5 es típico de cálculo, pero no al estilo de los usualmente propuestos en la escuela, cuyos elementos incluyen exclusivamente los datos que han de ser manejados para la solución. Por el contrario, la realidad es mucho más rica en información, lo que obliga a seleccionar los datos relevantes al propósito del ejecutor y desechar la información trivial o no pertinente.

¿Cómo ha sido abordado este problema-tipo? (ver tabla 5 y grafo 2 ).

El vector limite revela que, estabilizada la conducta, la mayor probabilidad de ser observado cualquier escolar corresponde a la actividad $\mathrm{E}_{5}$, seguida a corta distancia de la $E_{s}$ y con probabilidad decreciente $E_{1}$, $E_{9}$, etc... y que difícilmente se le encontrará en $E_{3}, E_{7}$, etc... Pero una vez leído el problema y situado en $E_{\text {s, }}$ su conducta siguiente será persistir en la misma con una

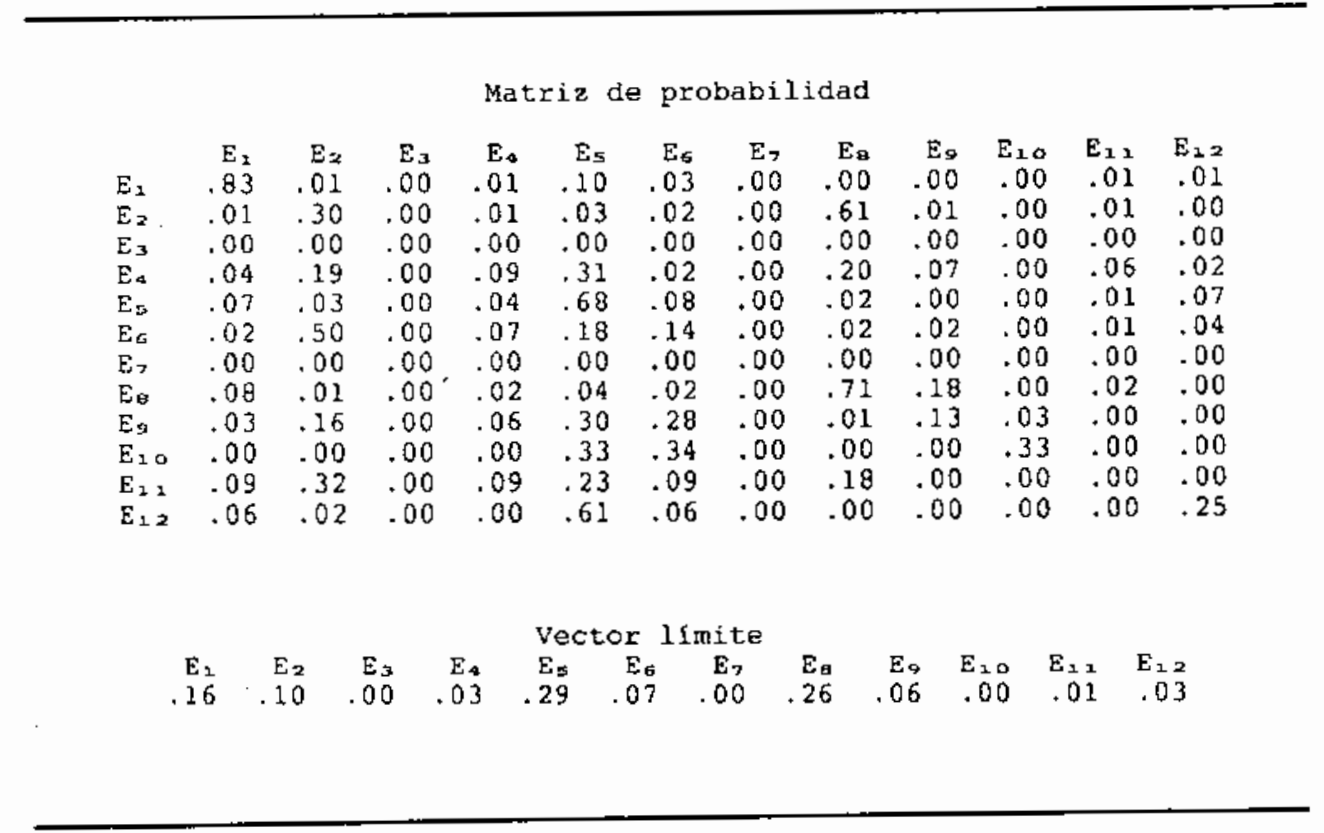

Grafo 2

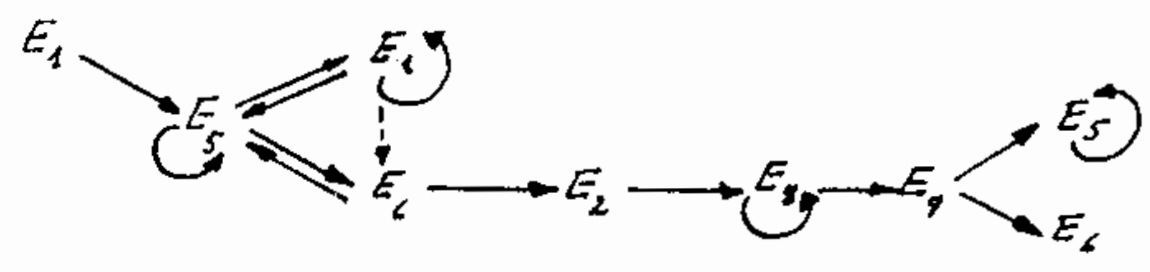


probabilidad de 0.68 . La abundancia de datos deja al escolar perplejo, se repite una y otra vez la pregunta, repasa de nuevo los datos, y trata con dificultad de encontrar la información pertinente, bien hacia $\mathrm{E}_{1}$ volviendo a leer el problema, bien hacia $\mathbf{E}_{6}$ para construirse un método que đirija su acción hacia el fin buscado. Situados en este punto, se avanza hacia el cálculo, persistiendo en él o saliendo hacia la resolución mediante una respuesta numérica. El hecho de que la fase siguiente no sea $E_{12}$-que indicaría que los alumnos dan por terminado el problema-, sino hacia Es o E6 señala bien a las claras que el resultado no les parece satisfactorio o que ensayan una nueva hipótesis, pero sin verificación operatoria ( $\mathrm{E}_{10}$ ) o estratégica $\left(\mathrm{E}_{11}\right)$.

\subsection{Estrategia generalizada}

El cálculo matricial permite además desvelar el uso de heurísticos y su secuencia en un colectivo de estudiantes, sea cual sea el tipo de problema. Es obvio que lo que se gana en generalidad se pierde en especificidad (niños concretos, problemas-tipo); sin embargo podría resultar provechoso conocer la evolución de los recursos mentales y operativos de los escolares de diferentes nivcles madurativos. Por lo que se refiere a nuestra muestra, los resultados son los de la tabla 6 .

Es evidente que la primera conducta del escolar es leer el problema. A partir de ahí, bien se lanza a calcular, $\left(\mathrm{E}_{8}\right)$ bien se sume en una fase de incertidumbre $\left(\mathrm{E}_{5}\right)$ según los dos estados más probables del vector límite.

Hay que destacar la semejanza del grafo 3 con la estrategia desarrollada en el problema número cinco. $\mathrm{Pa}$ rece claro que el mayor número de transiciones, sea cual sea el estado en que se encuentra el sujeto en el momento de ser observado, es hacia $E_{s}$; que esta situación es recurrente en buena medida, pero cuando se sale de ella es o porque los alumnos prevén un modo de ataque $\left(\mathrm{E}_{6}\right)$ o porque sin reflexión previa escriben datos; en ambos casos la cadena se desliza hacia las operaciones $\left(\mathrm{E}_{8}\right)$ y hacia el resultado numérico $\left(\mathrm{E}_{9}\right)$, para

Matriz global 15 problenas $x 20$ niñost

$\begin{array}{lllllllllllll} & E_{1} & E_{2} & E_{3} & E_{4} & E_{5} & E_{G} & E_{7} & E_{3} & E_{y} & E_{20} & E_{12} & E_{12} \\ E_{1} & .84 & .02 & .00 & .00 & .10 & .03 & .00 & .00 & .00 & .00 & .00 & .01 \\ E_{2} & .01 & .35 & .00 & .02 & .06 & .03 & .00 & .50 & .01 & .00 & .01 & .01 \\ E_{3} & .00 & .01 & .46 & .25 & .00 & .03 & .00 & .23 & .02 & .00 & .00 & .00 \\ E_{4} & .02 & .21 & .22 & .05 & .19 & .02 & .00 & .19 & .04 & .01 & .03 & .01 \\ E_{5} & .05 & .06 & .00 & .02 & .63 & .09 & .01 & .02 & .00 & .00 & .02 & .10 \\ E_{5} & .02 & .48 & .02 & .07 & .18 & .12 & .02 & .04 & .01 & .00 & .02 & .02 \\ E_{7} & .03 & .44 & .00 & .00 & .17 & .10 & .00 & .10 & .02 & .00 & .03 & .10 \\ E_{8} & .01 & .02 & .01 & .01 & .03 & .01 & .00 & .70 & .15 & .00 & .06 & .00 \\ E_{9} & .05 & .18 & .02 & .05 & .27 & .22 & .00 & .01 & .12 & .02 & .04 & .02 \\ E_{10} & .00 & .14 & .00 & .00 & .22 & .36 & .00 & .07 & .00 & .07 & .14 & .00 \\ E_{11} & .02 & .40 & .00 & .01 & .15 & .06 & .00 & .29 & .01 & .01 & .03 & .00 \\ E_{12} & .02 & .02 & .00 & .00 & .59 & .02 & .00 & .01 & .00 & .00 & .00 & .34\end{array}$

$\left.\pi(\infty)=\pi(32)=\frac{\text { Vector }}{1.12 \cdot 12.02} \frac{\text { limite }}{2.02} \frac{\text { dea }}{.24} \frac{\text { situacion }}{.06} \cdot \frac{\text { total }}{29} \cdot 00.00 .03 .04\right)$

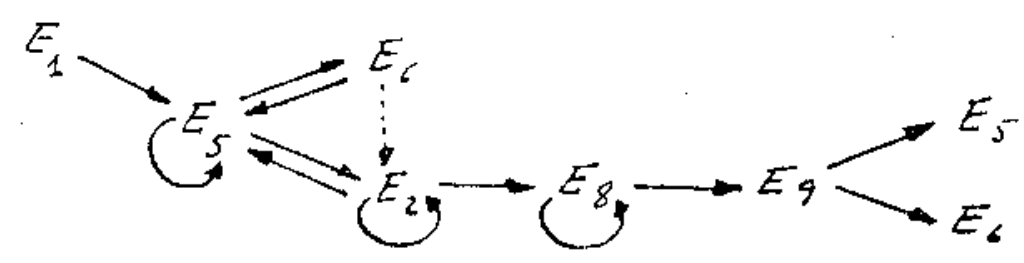


entualmente retroceder hacia $\left(\mathrm{E}_{s}\right) \circ\left(\mathrm{E}_{6}\right)$.

Es notable la existencia de un esquema transicional bastante constante y cuyo núcleo fundamental está constituido por los eslabones que requieren manipulación numérica. No es de extrañar tratándose de resolución de problemas matemáticos, pero se echan en falta estrategias no computacionales, como la representación, la formulación de planes, la estimación, la comprobación de resultados y la reordenación del proceso.

\section{CONCLUSIONES Y PROPUESTAS}

Una vez estabilizada la conducta después de n pasos, la probabilidad de observar al niño en uno cualquiera de los doce estados definióos en la resolución de problemas es la representada por el vector límite de la tabla 7 , cuyo orden es:

- E8, cálculos operatorios. El reduccionismo de la matemática al número conduce al niño a pensar que un problema es ante todo una combinación de números. Cualquiera que sea el modo de aborđar el problema, alcanzado el estado de equilibrio, en un $29 \%$ de los casos encontraremos a los alumnos de la muestra calculando, aunque no sepan exactamente para qué, conclusión parecida a la que habían llegado Carpenter y sus colaboradores (1980) al of recer los resultados de una amplia investigación en EE.UU. Si bien es cierto que el cálculo es un instrumento para la resolución de problemas numéricos, puede derivar hacia algo repetitivo y trivial que amenaza con ahogar el razonamiento. Convendría aumentar la propuesta de problemas sin contenido numérico, cuya solución sea exclusivamente verbal, gráfica o activa. (p.e. Bransford y Stein, 1986).

- Es, desconcierto. La perplejidad, el estado de tensión, de suspensión del ánimo, es la postura más natural ante un problema que se contempla por primera vez. El desconcierto no es malo, siempre que signifique el estado previo que suscita la organización de las operaciones mentales para salir de él. Lo que ocurre es que esta salida es el resultado de una anticipación o hipótesis que marca el camino que hay que recorrer después. Encontrarlo confiere al niño seguridad y confianza en sí mismo. No podemos, pues, abandonar al niño a su suerte y abocarlo a este estado de desconcierto. El hecho de que nada menos que un $24 \%$ de los estados en que podemos encontrar a un sujeto sea en éste de perplejicad, demuestra que los probłemas, más que obstáculos que hay que remontar inteligentemente, son cárceles emocionales en las que el niño se encuentra prisionero. Es preciso dirigir el proceso con oportunas preguntas que conduzcan a la búsqueda de alguna senda para empezar a caminar con la ilusión que despierta un razonamiento equilibrado. Desde nuestro punto de vista ésta es una fase en la que la intervenvión es más necesaria, no tanto para dar instrucciones, cuanto para sugerir claves o pistas que actuando a modo de uorganizadores» de la información permitan reconducir el proceso cognitivo y salir del «impasse». Buena parte de la bibliografia citada ofrece recursos y estrategias útiles a este efecto.

- $\mathrm{E}_{1}$, lectura del problema. Parece obvio que la lectura comprensiva del problema sea la condición previa para su resolución. Sin embargo, la inveterada costumbre del profesorado de invitar a reiteradas lecturas cuando el niño manifiesta alguna dificultad, conduce a éste a volver una y otra vez a la lectura, como si ésta fuera por si sola capaz de despejar las nubes de la mente. La lectura por la lectura es una acción estéril, a menos que sea la plataforma a partir de la cual se entienda la situación, se inquiera el propósito, se extraigan los datos y se perciba un camino razonable desde la pregunta hasta la solución.

- $E_{2}$, escribir datos. Es cierto que para calcular hay que escribir previamente los datos (en nuestra matriz la transición $\mathrm{E}_{2}-\mathrm{E}_{8}$ es de $50 \%$ ), pero no es normal que la transición $\mathrm{E}_{2}$ sobre sí misma sea del $35 \%$, como si de la mera escritura de datos surgiera la luz resolutiva. Por otro lado, que el vector límite contemple $\mathrm{E}_{2}$ como un estadio posible en el $12 \%$ de las ocasio. nes en que el niño puede ser observado, evidencia el carácter mitico que se concede al número en sí mismo, por encima del propio proceso heurístico. Es preciso, pues, estimular al niño a descubrir el porqué y el para $q u e ́$ de la escritura y el cálculo de datos.

- $\mathrm{E}_{6}$, formulación de hipótesis. Su baja incidencia $(6 \%)$ pone de relieve que los niños atacan los problemas sin un plan preconcebido: leen sin saber lo que tienen que buscar en el enunciado, y escriben datos y calculati sin una regla o hilo conductor que una la pregunta con el resultado y dé consistencia a los distintos pasos intermedios. Sin embargo, el hecho de que, decidido un plan de acción, la cadena se deslice: $E_{6}-E_{2}$ - $\mathrm{E}_{8} \rightarrow \mathrm{E}_{9} \rightarrow$, es un indicador de que la producción de planes de trabajo y la formulación de hipótesis restrin- 
gidas (《si combino tales datos, obtengo tales otros») debe ser una estrategia prescriptiva para el profesor, dado que una de las características de los buenos resolutores es la de conjeturar, hacer predicciones y someterlas a prueba (Grupo Cero, 1987, p. 94).

Con ser esta producción mental el núcleo del pensamiento inteligente, sin embargo está totalmente abandonado en la enseñanza, y lo más sorprendente es que lo esté en el campo de la matemática, cuyos cultivadores hacen gala de proceder racionalmente en su discurso. (Schoenfeld, 1985a, p. 27).

- E9, resolución. No es lo mismo calcular que resolver. Calcular es combinar números de acuerdo con ciertas reglas; resolver es dar respuesta coherente a la cuestión suscitada por el problema; por eso muchos estudiantes no se sorprenden de los resultados disparatados de sus cálculos, porque no unen el resultado numérico con el interrogante conceptual. De ahí que los alumnos de nuestra muestra en vez de progresar hacia $E_{10}$, que parece el camino razonable antes de rectificar, lo han hecho hacia Es, sorprendidos de la eventual inconsistencia de la solución, o hacia $E_{6}$ para intentar un nuevo proceso. Cuando el ciclo se reitera sin rectificación razonable, se entra en una dinámica de ensayo y error muy discutida como estrategia fundamentada en la resolución de problemas. Este aspecto puede ser abordado en especial mediante matrices de transición y arrojar alguna luz sobre resultados de la investigación, pues mientras algunos trabajos afirman el uso restringido del ensayo y error entre los buenos resolutores, en otros se ha descubierto que lo utilizan con una frecuencia superior a la media. Pero hallazgos parecidos se han encontrado entre los malos resolutores. Descubrir desde qué heuristicos previos vienen y hacia cuales derivan, puede ayudar a clarificar to que hay detrás de la pura constatación cuantitativa.

- E12, bloqueo o ausencia de conducta. Difiere de $E_{s}$, en que aquel desconcierto conduce a formularse de nuevo la pregunta, pedir más datos, volver a leer el problema, etc., mientras que $E_{12}$ es el estancamiento o abandono por no saber seguir. Sin embargo, es notable que los niños permanezcan tan poco tiempo en este estado. Hay como una especie de compulsión a salir de él, por lo que debería ser ayudado, como en $E_{5}$, hacia el que existe la mayor probabilidad de desplazamiento, bien para formular hipótesis, bien para descubrir el núcleo conceptual del interrogante.

- E11, rectificación. La carencia de una estrategia válida que dirija la acción tiene como resultado que el estudiante ensarte operación tras operación hasta obtener un dato que se le aparezca como solución, sin que las fases intermedias signifiquen nada para él. Por eso la rectificación es tan improbable, $y$, cuando aparece, es para volver a escribir nuevos datos o para corregir errores de cálculo más que para reordenar el método. Incluso cuando se percibe la solución como absurda, vuelve con dificultad al principio para rectificar el dis- curso, más bien suele pasar a $\mathrm{E}_{12}$, quedando desilusionado, pero inactivo.

- $\mathrm{E}_{4}$, utilización de datos irrelevantes. Decir que los prob lemas responden al medio vital del niño es mentira; quizá respondan en su contenido, pero no en la forma. Los problemas escolares reflejan una realidad «descafeinada», limpia de impurezas, sólo con los datos necesarios para las operaciones, como ya se ha señalado. Pero èsta no es la realidad; por el contrario, en ella tiene que aprender el niño a seleccionar, entre muchos datos, aquéllos pertinentes a su situación, o buscar información que le es necesaria y que no encuentra de forma inmediata. De ahí que los buenos problemas deberían incluir datos irrelevantes y carecer de alguno imprescindible. Ello forzaria al niño a pensar, a analizar la situación más que aplicar unas rutinas de cálculo, a veces carentes de sentido.

- E3, representación de datos. Es preocupante el hecho de que los niños apenas utilizan un sistema de representación, limitación muy ligada a la falta de un diseño anticipado del proceso. Es preciso estimular esa actividad con el trazado de figuras, esquemas, itinerarios o trayectorias, formas, etc... que sitúen al estudiante entre lo intelectual y lo material, entre lo inteligible y lo sensible, y contribuya a apoyar intuitivamente la secuencia prevista. La literatura vuelve a poner de relieve que la instrucción específica en representación de datos mejora la solución de problemas presentados gráficamente, aunque no produce una mejora significativa en la habilidad resolutora general (Nelson, 1975).

- $\mathrm{E}_{7}$, anticipación de los resultados. No se trata, evidentemente, de adjininar o pronosticar un dato numérico, sino de dar razón sobre el sentido del resultado. Solamente anticipando la dirección de la respuesta puede el atumno percibir la adecuación o no de la solución. La carencia absoluta de esta producción (probabilidad cero en este estudio) es la que permite aceptar como razonables, resultados absolutamente absurdos. No es de extrañar esta dura constatación, pues como ha puesto de relieve Edwards (1984), estudios comparados muestran que los textos de matemáticas en varios países dedican a la estimación menos de $3 \%$ de su contenido.

Sería deseable una intensificación de la práctica de estimación pues, como ya había constatado Hall (1977), existe una estrecha relación entre la capacidad para hacer estimaciones y la habilidad para resolver problemas. Más recientemente, el mismo Edwards, y entre nosotros Segovia (1986), han demostrado que la ejercitación específica mejora esta competencia, y aunque no se haya demostrado que a corto plazo perfeccione la aptitud general para la resolución de problemas parece ser que ésta sólo aparece posteriormente una vez que las habilidades fragmentarias se integran en un esquema más general.

- E10, comprobación. Es muy grave el hecho de que 
una actividad tan importante para el desarrollo de la capacidad intelectual en el campo de la resolución de problemas alcance una probabilidad nula de que aparezca en el estado de equilibrio. Los alumnos que llegan a un resultado numérico, sea el que sea, y se despreocupan de si es correcto o no, no pueden conseguir satisfacción alguna, ni gustar ese placer intelectual de haber llegado a conocer sensorial, funcional y tensionalmente lo desconocido, y que constituye un sistema absolutamente económico de refuerzo positivo.

La conclusión actuacional de este trabajo es que si después de una lectura inteligente, conseguimos que nuestros alumnos formulen espontánearnente hipótesis razonadas, dibujen el plano de la situación y comprue.

\section{REFERENCIAS BIBLIOGRÁFICAS}

ALONSO, V., 1986, Estrategias operativas en la resolución de problemos. Tesina de Licenciatura (inédita). (Departamento de Estadistica e Investigación Operativa. Universidad: Granada).

ANGUERA, M.T., 1983, Manual de prácticas de observación. (Trillas: México)

BARNET, J., VOS, K. and SOWDER, L., 1979, A Review of Selected Literature in Applied Problem-Solving Research, en LESH, R., MIERKIEVICZ, D. and KANTOVSKI, M. (Eds), Op. cit. 73-109.

BILLSTEIN, R., LIBESKIND, S. and LOTT, J.W., 1984, A Problem-Solving Approach to Mathematics for Elementary School Teachers. (The Benjamin/Cummings Publishing Company Inc., Melo Park: California).

BRANSFORD, J.D. and STEIN, B.S., 1986, Solución IDEAL de problemas. (Labor: Barcelona).

BROWN, S.I., 1983, La resolución de problemas y la formación docente: El humanismo entre modelos y confusiones. Estudios en Educación Malemática, 3, pp. 9-46.

CARPENTER, T.P. et at., 1980, Results of the Second NAEP Mathematics Assesment: Secondary School, Mathematics Teacher, Vol. 73, 5, pp. 329-338.

CARSS, M., (ed.), 1986, Proceedings of the fitht International Congress on Mathematical Education. (Birkhäuser Boston Inc.: Brisbane).

CHARLES, R.I., 1982, An Instructional System for Mathematical Problem Solving, en RACHLIN, S. (Ed.), Op. cit., pp. 17-32.

CHRISTIANSEN, B. et al., 1986, Research and Teaching, en CARSS, M. (Ed.), Op. Cit., pp. 285-292.

EDWARS, J., 1984, Computationai Estimation for Numeracy, Educational Studies in Mathematics, 15. ben si lo hipotético, planificado y resuelto es lo correc. to, habremos establecido las bases sobre las que se apoya operativamente la resolución de problemas matemáticos, tal como se ha evidenciado por las cadenas conductuales, los eslabones que las constituyen y las vias alternativas por las que fluye el proceso resolutivo.

Pero para esto sería necesario que en las Escuelas del Profesorado y Facultades se abordara con el mismo cri. terio de racionalidad la solución de problemas, porque los métodos con que los futuros profesores enseñen no serán los que aprendieron en los libros, sino más pro. bablemente los que aprendieron a través de los propios métodos con que ellos mismos fueron enseñados.

FELLER, W., 1973, Introducción a la Teoria de la Probabilidad y sus aplicaciones. (Limusa Wiley: México).

FIELKER, D.S., 1985, Siete estrategias para plantear problemas en geometría, La enseñanza de la Matemática a debate, M.E.C., Madrid, pp. 97-109.

FLAHERTY, E.G., 1975, The Thinking Aloud Technique and Problem-Solving Ability, Jownal of Educational Research, 62, pp. 223-225.

FRANCÉS, M.P., GONZÁlEZ, A. y SÁENZ, O., 1987, Naturaleza funcional de las relaciones entre variables conductuales asociadas a una secuencia temporal. Thales, 6 , pp. $77-88$

FRANCÉS, M.P. y SÁENZ, O., 1987, Descripción de la conducta dependiente en el párvulo mediante matrices de transición. Revista de Educación de la Universidad de Granada, 1 .

GIMENO, J., 1981, Teoria de la enseñanza y desarrollo del curriculo. (Anaya: Madrid).

GONZÁLEZ CARMONA, A., 1975, Algoritmo de clasificación de matrices de transición en una cadena markoviana finita. Cuadernos del Departamento de Estadística Matemática, Universidad de Granada, 1, pp. 32-39.

GRUPO CERO, 1987, De 22 a 16. Un proyecto de curriculo de Matemáticas. (Mestral Libros: Valencia).

HALL, W.D., 1977, A Study of the Relationship between Estimation and Mathematical Problem-Solving among Fifth Grade Students, Dissertation Abstracts International, 37, 6324A-6325A.

HOWSON, G., y MALONE, J., 1986, Curriculum Development, en CARSS, M. (Ed.), Op. Cit., pp. 187-196.

KANTOVSKI, M.G., 1977, Processes Involved in Mathematical Problem Solving, Journal for Research in Mathematics Education, 8, pp. 163-180. 
KRULIK, S., y REYS, R. (Eds.). Problem solving in school mathematics: 1980 Yearbook. (National Council of Teachers of Mathematics. Reston. Virginia).

LEE, K.S., 1982, Leading Problem Solving in an Elementary School Classroom cn RACHLIN, S. (Ed.) Op. cit. pp. 44-48.

LESH, R., MIERKIEVICZ, D, y KANTOVSKI, M. (Eds.) 1979, Applied Mathematical Problem Solving, (College of Education, The Ohio State University: Columbos, Ohio).

LESH, R., LANDAU, M. y HAMILTON, E., 1983, Conceptual Models and Applied Mathematics ProblemSolving Research, en LESH, R. and LANDAU, M. (Eds.): Adquisition of Mathematical Concepts and Processes, (Academic Press: New York).

LESH, R., NISS, M. y LEE, D., 1986, Applications and Modeling, en CARSS, M. (Ed.), Op. Cit., pp. 197-211.

LESTER, F.K., 1982, Issues in Mathematical ProblemSolving Research, en RACHLIN, S. (Ed.), Op. cil., pp. 141-147.

MEANS, G.H. y LOREE, M.R., 1986, The Influence on Problem-Soiving Ability of Following an Efficient Model, Journal of Educational Research, 62, pp. 135-141.

NELSON, G.T., 1975, The Effects of Diagram Drawing and Translation on Pupil's Mathematical Problem-Solving Performance, Dissertation Abstracts International, 35, 4149A.

\section{APÉNDICE}

1. Cuando salgo de paseo los domingos puedo it andando, en bicicleta o en coche. Visito a mis amigos que viven en la calle Larga, calle Mayor, calle del Sol y calle Zapatería. La calle Larga, como indica su nombre, es la que tiene mayor longitud, la más corta es la calle del Sol y las otras dos son intermedias y miden lo mismo. La calle del Sol es perpendicular a las calles Larga y Zapateria, la calle Mayor es paralela a la primera. ¿De cuántas maneras distintas puedo realizat mis visitas?

2. He visto o estudiado que un pájaro tiene 2 patas, un perro tiene 4 , una abeja tiene 6 , una araña tiene 8 , un cangrejo tiene 10 , un ciempiés tantas como indica su nombre. Un perro es mayor que un cangrejo y éste mayor que la araña y el ciempiés. En un mismo lugar se encuentran reunidos 2 perros, 1 abeja, 1 araña, 1 congrejo y 7 ciempiés. Si yo cuento en base 2 , ¿cuántas patas tiene el pájaro?, ¿cuántas patas tiene la araña? Si cuento en base 5 , ¿cuántas patas tiene el cangrejo?, ¿cuántas tiene la abeja?

3. Al empezar las clases mis hermanos y yo necesitábamos libretas, bolígrafos, rotuladores, compases, reglas, 1 caja de lápices de color y otra de acuarelas. Recuerdo que 17 libretas nos costaron 1.105 pesetas, 4 cajas de acuarelas 900 pesetas, 70 pesetas un par de bolígrafos, 200 pesetas 3 rotuladores y 1 regla. Al salir a la calle me encontré con un amigo que me preguntó: ¿cuánto cuesta cada libreta?, ¿cuánto me costarán 9 libretas? Yo le respondi: una libreta cuesta y 9 libretas te costarán
POLYA, G.r 1945, How to solve it. (Princeton University Press: Princeton).

RACHLIN, S. (Ed.), 1982, Problem solving in the Mathematics Clossroom. MATH. Monograph 7. Mathematics Council of the Alberta Teachers Association.

RUDNICK, J.A. and KRULIK, S., 1982, Problem Solving: Goals and Sirategies, en RACHLIN, S., (Ed.), Op. cit., pp. $17\lfloor-175$.

SCHOEN, H.L. y OEHMKE, T., A New Approach to the Measurement of Problem Solving Skills en KRULIK, S. and REYS, R. (Eđs.): Op. cit. pp. 216-227.

SCHOENFELD, A.H., 1980, Heuristics in the Classroom, en KRULIK, S. and REYS, R. (Ed.), Op. cit., pp. 9-22.

SCHOENFELD, A.H., 1985a, Ideas y tendencias en la resolución de problemas. La enseñanza de la Matemática a debate. Servicio de publicaciones del M.E.C., Madrid, 25-30.

SCHOENFELD, A.H., 1985b, Sugerencias para la enseñanza de la resolución de problemas matcmáticos. En La enseñanza de la Matemática a debate. Servicio de publicaciones del M.E.C., Madrid, 31-65.

SEGOVIA, 1., 1986, Estimación y cálculo aproximado en la E.G.B., Memoria de Licenciatura (Inćdita), Departamento de Didáctica de la Matemática, Universidad de Granada. Granada.

SILVER, E.A., 1977, Student Perceptions of relatedness among mathematical word problems. Dissertation Abstracts International, 1977, 38, 5438A.5439A.

4. Fui a una confitería para comprar golosinas. Tenía un biIlete de 1.000 pesetas, 2 de 500,3 monedas de 100 pesetas y 75 pesetas el kilo, bombones a 245 pesetas los 100 gramos, pastelitos a 495 pesetas el medio kilo, pasteles a 50 pesetas cada uno, almendras rellenas de turrón a 200 pesetas los 200 gramos. Compré 300 gramos de bombones y 300 gramos de almendras y 12 pasteles. ¿Cuánto dinero dejé en la confitería?

5. Es la fiesta del colegio. El Director encargó de decorar el salón de actos a todos los alumnos de la clase que somos 30: 7 de 8 años, 8 de 9 años y 15 de 10 años. EI escenario tiene las medidas que indica el dibujo, con tres puertas de entrada y salida. Queremos rodearlo con volante de papel rojo y cruzarlo por el techo de esquina a esquina con cintas de papel azul. El papel rojo cuesta a 150 pesetas el metro, el amarillo 175 pesetas y el verde 200 pestas. Tencmos además 2 rollos de cinta azul, 3 de cinta violeta y 4 de cinta dorada. ¿Cuánto nos costará là decoración?

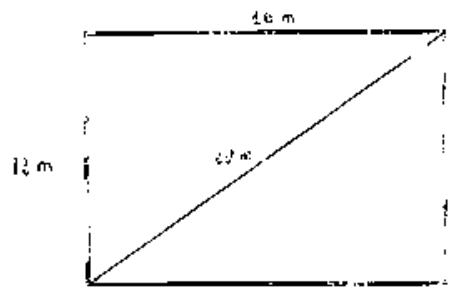

ENSEÑANZA DE LAS CIENCIAS, 1988, 6 (3) 